\title{
Empirical Evaluation of Semantic Alignment Quality Metrics for Vehicle Domain Component Frameworks Interface Ontologies
}

\author{
Sangita De, ${ }^{1}$ Juergen Mottok, ${ }^{2}$ Premek Brada ${ }^{3}$ and Michael Niklas ${ }^{4}$ \\ Continental AG, Germany, ${ }^{1,4}$ OstBayerische Technical Hochschule (OTH), Germany, ${ }^{2}$ University of West Bohemia, Czech Republic ${ }^{1,3}$ \\ sangita.de@ outlook.de, ${ }^{1}$ juergen.mottok@oth-regensburg.de, ${ }^{2}$ brada@ kiv.zcu.cz, ${ }^{3}$ michael.niklas@ continental-corporation.com ${ }^{4}$
}

\begin{abstract}
Semantic alignment of application software components' ontologies represents a great interest in vehicle application domains that manipulate heterogeneous overlapping knowledge application frameworks. In the past few years, with the growth in the novel vehicle service requirements such as autonomous driving, V2X (Vehicle-to-Vehicle communication) and many others, automotive application software component models are becoming increasingly collaborative with other qualified cross-enterprise industrial partners to accomplish these complex service requirements. The most daunting impediment to this cross-enterprise collaboration is semantic interoperability. For efficient services collaboration through cross-enterprise semantic interoperability between the vehicle application frameworks' software components, aligning the interface ontologies of these components by identifying the depth of semantic alignment relationships between the concepts of the interface ontologies is the major focus of this paper. In contrast to several existing ontology structural metrics, this work defines, evaluates and validates ontology metrics to measure the depth of semantic alignment between the vehicle domain software component frameworks' interface ontological models. To emphasize the substantial role of semantic alignment of software component frameworks' interface ontologies in semantic interoperability, a typical vehicle domain case study involving vehicle applications is considered for demonstration.
\end{abstract}

\section{Introduction}

Enriching ontology mapping with semantic relations has been the focus of a large amount of research that led to a broad range of techniques to discover the corresponding or semantically matching concepts between ontologies. Scientific techniques or metrics evaluating the semantic quality and the validity of an ontology are necessary to be determined for ontology engineering process and for an enduser to better understand whether a given ontology is suitable for his application domain (Arnold and Rahm 2014).

Copyright (C) 2020, Association for the Advancement of Artificial Intelligence (www.aaai.org). All rights reserved.
In the vehicle domain, from a modeling perspective there can be an overwhelming number of ways on how to implement even a simple two-way communication using legacy or open-source platforms' Software Components (SWCs) frameworks' interfaces. Due to the presence of several enterprise platform-specific frameworks' interfaces and their vocabularies of concepts, results in a source of discord in understanding of the meaning of these concepts by experts from other knowledge domain platforms. Most semantic alignment approaches in vehicle domain address this problem by semantically mapping the vocabularies of platform-specific interface concepts based on synergies in concepts. To ease the semantic alignment and semantic interoperability between the heterogeneous platformspecific vocabularies of interface concepts, ontologies are used for schematic representation of SWCs' interface metamodels' specifications. This work considers W3C standardized ontology language OWL2 (Web Ontology Language version 2.0) as a metamodeling language to describe SWCs' interface ontologies as metamodels.

In principle, evaluation of semantic alignment quality for the vehicle SWC frameworks' interface ontologies using metrics is substantial to guarantee that it meets the vehicle application domain requirements for crossenterprise semantic interoperability (Breitman, Felicíssimo and Cysneiros 2003). Evaluation of semantic alignment between various SWCs' interfaces ontologies using semantic-aware ontology metrics are stable regarding possible additions of further axioms to a given interface ontology, in the context of frequently changing automotive application SWCs specifications (Vrandecic and Sure 2007).

\section{Contribution}

Given that plethora of multiple ontologies representing the vehicle domain SWC frameworks' interfaces may present different types of heterogeneity (terminological, structural or semantic, among others), we need promising techniques to correctly discover mappings between their entities (Sil- 
va, Revoredo, Baião, and Euzenat 2020). In this scenario, using a platform-independent, domain-specific Ontology mediator for solving any terminological mismatch between semantically equivalent concepts that can appear with different frameworks' SWCs interface ontologies within the vehicle domain, could sound a promising technique. However, in this research direction, the question that emerges is what is the depth of semantic alignment quality that can be achieved between the SWC frameworks specific interface ontologies, when using a generic, platform agnostic, domain-specific Ontology mediator. This contribution works towards addressing this question.

This work defines, evaluates, and validates the semantic alignment quality metrics for different frameworks' interface ontologies based on the analysis of TBox and ABox axioms (asserted and inferred), evaluated manually by domain experts based on the inferred artifacts obtained using inference rules (like Semantic Web Rule Language, SWRL) given to an automated reasoner as a part of the ontology framework. The inferred artifacts were validated by using the extended reasoner support to SPARQL query. This work measures the depth of the quality of semantic alignment between the cross-enterprise vehicle domain SWC frameworks' interface ontologies by measuring the semantic similarity (" $i s-a$ ") relationships between their concepts. Additionally, a typical vehicle domain case study was considered as a part of the evaluation approach.

\section{Background and Related Work}

The semantic of a SWC's interface concerns with the meaning of interface concepts specified by the requirements. In general, the semantic of vehicle applications SWC frameworks' interface ontologies fundamentally must include the following constructs (Feld and Müller 2011):

- Concepts: They are a prototype of a knowledge instance. In current scope, concepts can be modeled as ontology schema classes and subclasses representing vehicle application fundamental interface types like operation-based (for example, methods-invocations), event-based (for example, Publish-Subscribe), broadcast, data-passing (for example, SenderReceiver), etc.

- Is-a relation: A relation between concepts through which they can inherit from other concepts using this relation. For example, ClientServerOperation() concept "is- $a$ " or a type of method-invocations concepts.

- Has-part relations: This is the predominant type of relation between concepts. The semantics are that one concept is a child of another concept. For example, each SWC class "has", or a subclass named Ports.

- Properties. Pointers to plain values that do not have an identity, but their type are only evaluated with respect to the concepts to which they belong. For example, at- tributes types for concepts like methods-invocations can be representing Parameters types.

Often ontology metrics so far defined for automotive domain ontologies are structural based. Automotive ontology at the core of a car's information system as described by authors (Feld and Müller 2011) mainly contributes towards a reference ontological model design, highlighting vital areas of automotive application domain knowledge in conjunction with reasoning. However, the work does not describe ways to evaluate the quality of semantic alignment between the collaborating services considered within the given automotive ontology. The Ontology Alignment Evaluation Initiative (OAEI) is a coordinated international initiative whose one of the goals is to assess the strengths and weaknesses of ontology matching systems. Alin, an interactive ontology matching approach (Silva, Revoredo, Baião, and Euzenat 2020) using domain experts' feedback, is ranked as the highest data precision approach under OAEI. The mappings techniques used by Alin are concept and attribute mapping, relation mapping, etc. The OntoQA framework (Tartir, Arpinar and Sheth 2010) defines the quality of a populated ontology as a set of five schema quality features and nine knowledgebase (or instance-base) quality features. All the metrics defined by OntoQA framework, however, fail to define if they are structurally or semantically defined which is a common lapse. OntoClean (Guarino N. and Welty 2002) provides means to derive measurable mismatches of a taxonomy with respect to an ideal structure which considers the semantics of the " $i s-a$ " relationship. The mismatches described in this paper are mainly of structural nature.

\section{Ontology Metrics for Interface Semantic Alignment Quality Evaluation}

Metric-based techniques to evaluate ontologies offer a quantitative perspective of ontology quality. These techniques scan through a given ontology to gather different types of statistics about the knowledge presented in the ontology (Tartir, Arpinar and Sheth 2010). This contribution considers two categories of metrics for evaluation of semantic alignment quality between vehicle SWCs' interface ontologies: firstly, the schema metrics that address the semantics of interface ontology schemas and secondly, the instance metrics that address the semantics of instances organized within the interface ontology schemas.

\section{Semantic Similarity Schema (SSS) Metric}

Definition: This Schema metric indicates the richness, depth, and inheritance of an ontology schema. For a SWC's interface ontology schema, say $\mathrm{O}_{\mathrm{A}}, S S S$ metric can be interpreted and evaluated as the fraction or percentage of the number of classes $\left(E_{Q C}\right)$ of the schema $\mathrm{O}_{\mathrm{A}}$ that are semantically equivalent to the classes of other SWCs interface ontology schemas compared to the total number $\left(T_{R}\right)$ 
of the existing classes in the schema $\mathrm{O}_{\mathrm{A}} . T_{R}$ may include inheritance classes $\left(I H_{C}\right)$, noninheritance classes $(P)$ (these classes are not subclasses of other class in a given schema) and semantically equivalent classes $\left(E_{Q C}\right)$ in the schema $\mathrm{O}_{\mathrm{A}}$

$S S S=E_{Q C} / T_{R} \quad \ldots(1)$

Where $T_{R}=E_{Q C}+I H_{C}+P$ in (1).

\section{Instance Relationship Richness (IRR) Metric}

Definition: Instance metrics generally describes the knowledgebase. IRR Metric for a SWC's interface ontology schema, say, $\mathrm{O}_{\mathrm{A}}$, is defined as the percentage or ratio of the number of semantically similar or sameAs instances $\left(I_{S A}\right)$ of classes of the schema $\mathrm{O}_{\mathrm{A}}$ compared to the total number of instances of classes $\left(T C_{\text {Inst }}\right)$ present in the given schema $\mathrm{O}_{\mathrm{A}}$. TC Inst can include sameAs and differentFrom $\left(I_{D F}\right)$ classes' instances.

$I R R=I_{S A} / T C_{\text {Inst }} \quad \ldots(2)$

Where $T C_{\text {Inst }}=I_{S A}+I_{D F}$ in (2).

\section{Class Instance Connectivity (CIC) Metric}

Definition: CIC metric for an SWC's interface ontology schema, say, $\mathrm{O}_{\mathrm{A}}$, is defined as the ratio or percentage of the number of sameAs instances of semantically equivalent classes $\left(E Q_{i}\right)$ of the schema $\mathrm{O}_{\mathrm{A}}$ compared to the total number of semantically equivalent classes $\left(T_{Q C}\right)$ that are present in the interface ontology schema $\mathrm{O}_{\mathrm{A}}$. The $C I C$ metric for an ontology schema $\mathrm{O}_{\mathrm{A}}$, fundamentally represents the proportion of class equivalence relationships that are being utilized by the class' instances $\mathrm{O}_{\mathrm{Ai}}$ at instance level to create sameAs semantic relationships. Value evaluated for $C I C$ metric indicates class to class connectivity and ensures the data in the knowledgebase represents most of the knowledge in the schema (Tartir, Arpinar and Sheth 2010).

$C I C=E Q_{i} / T_{Q C}$

Where $E Q_{i} \propto$ (sameAs REL $\left.\left(\mathrm{O}_{\mathrm{Ai}}\right)\right)$ in (3).

\section{Methodology for Evaluation and Validation of Interface Semantic Alignment Quality Metrics}

Guided by literature artifacts (Staab, Walter and Parreiras 2014) in the past, the semantic alignment between concepts of platform specific ontological models can be simplified by using a generic, platform-agnostic, domain-specific ontological model as an ontology mediator between different framework specific interface ontological models. To simplify the manual evaluation and validation approach of semantic alignment metrics, a typical vehicle domain case study has been considered as part of the current research work. With the considered case study, the quality of semantic alignments between the heterogeneous SWC frameworks' interface ontologies are manually evaluated, based on observation of the inferred artifacts generated by an automated reasoner on semantic similarity (" $i s-a$ ") relationships like equivalence, sameAs, etc. with the help of proper SWRL rules constructed for the same. The depth of semantic alignments between the frameworks' interface ontologies within the considered vehicle domain case study are evaluated in terms of percentages of the given interface semantic alignment quality metrics. Protégé, an opensource ontology development tool with a plug in support of a reasoner (Pellet) has been considered in the current work for the design of SWCs' interface ontologies using OWL2.

\section{A Vehicle Domain Case Study}

The vehicle domain case study considered for the illustration of semantic alignment quality metrics is Collision Avoidance service from Advanced Driver Assistance System (ADAS) functional domain. This vehicle case study demands efficient collaboration of services between heterogeneous SWC frameworks, as service providers. Based on our earlier illustration on semantic synergies explored between different heterogeneous SWC frameworks' interface metamodel entities (De, Niklas, Rooney, Mottok and Brada 2019), it can be presumed that the semantic traits of the various vehicle SWC frameworks' interface metamodels that has semantic commonality among majority of the given interface metamodels must be abstracted as a generic, platform-independent, domain-specific interface semantic trait for vehicle application SWCs. A collection of such generic interface semantic traits is considered in composition of an ontology mediator.

An ontology mediator, DM, is a platform and technology agnostic ontology, and therefore can be used as common semantic background to glue the various semantic concepts of heterogeneous SWC frameworks' interface models when represented as ontologies. Along with an automated reasoner support of the ontology framework, the DM ontology, therefore, helps in semantically mapping between the concepts of heterogeneous frameworks' interface ontologies based on inferred artifacts, as also illustrated in Fig. 1. The given case study includes interface ontologies of SWC frameworks, namely, AUTOSAR Adaptive (as Source 1) from automotive knowledge domain, Franca (as Source 2) from infotainment knowledge domain and ROS2 (as Source 3) from robotics knowledge domain, as illustrated in the Fig. 1.

\section{Evaluation of SSS Metric for Interface Ontology Sources}

The SSS metric is evaluated for each of the given interface ontological model sources represented as schemas, that is, Source 1, Source 2 and Source 3 in Fig. 1. 


\begin{tabular}{|c|c|c|c|}
\hline 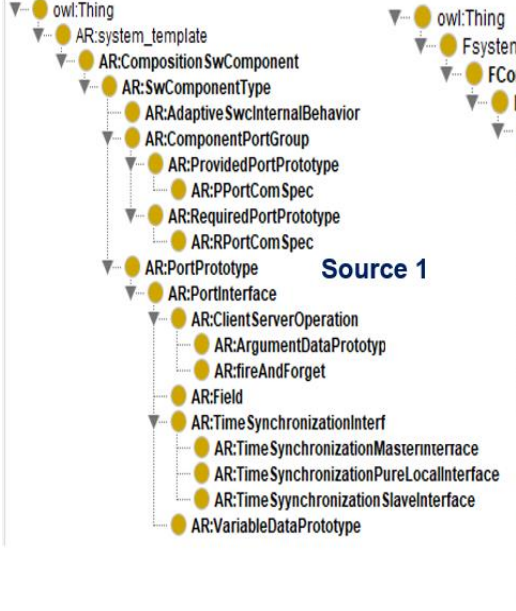 & $\begin{array}{l}\text { n_Template } \\
\text { mpositionComponentPrototype } \\
\text { FComponentPrototype } \\
\text { FPortPrototype } \\
\text { FPortinterface } \\
\text { FAttribute Source } 2 \\
\text { FBroadcast } \\
\text { FMethod } \\
\text { FArgumentDataPrototype } \\
\text { FrgumentMappingType } \\
\text { FRERAL_METHOD } \\
\text { FrovidedPortPrototype } \\
\text { FRequiredPortPrototype }\end{array}$ & $\begin{array}{l}\text { ROS:Node } \\
\text { ROS:Parameter Source } 3 \\
\text { ROS:Service } \\
\text { ROS:ServiceClient } \\
\text { ROS:ServiceServer } \\
\text { ROS:Topic } \\
\text { ROS:Data_Publisher } \\
\text { ROS:Data_Subscriber } \\
\text { ROS:Value } \\
\text { ROS:Datatypes } \\
\text { Float } \\
\text { Int } \\
\text { String }\end{array}$ & 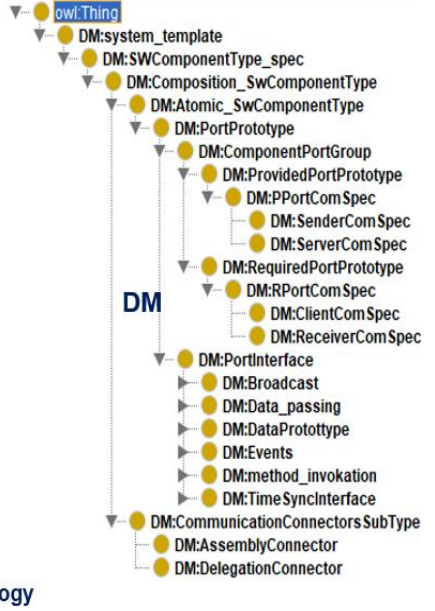 \\
\hline
\end{tabular}

Fig.1. Abstract representation of case study using heterogeneous SWC framework interface metamodels as ontologies.

In consideration to the interface ontology sources in Fig.1, the semantic similarity relationships that are determined by the percentage of semantic equivalence relationships that are explicitly expressed between the interface ontology schemas' classes and subclasses using asserted axioms and inferred equivalence class axioms (TBox axioms) generated by the reasoner. Fig. 2 and Fig. 3 illustrates measurement of percentage of semantic equivalence class axioms for each of the given interface ontology sources. The inferred semantic equivalence class axioms for the given interface ontology schemas are automatically generated by the reasoner of the ontology framework. As observed from Fig. 3, Source 3 expresses the maximum percentage of semantic equivalence relationships at schema level.

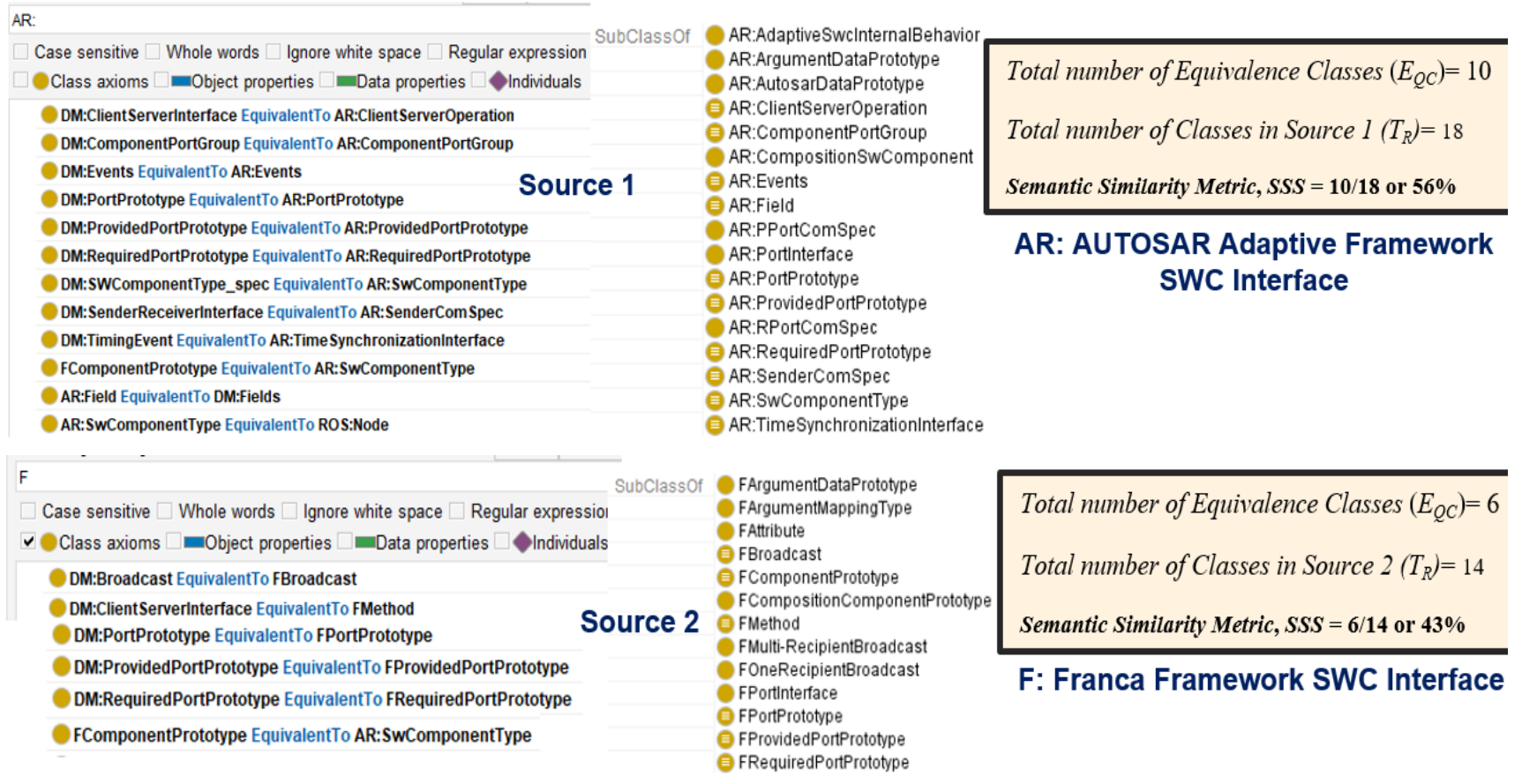

Total number of Equivalence Classes $\left(E_{Q C}\right)=10$
Total number of Classes in Source I $\left(T_{R}\right)=18$
Semantic Similarity Metric, SSS $=\mathbf{1 0} / \mathbf{1 8}$ or $\mathbf{5 6} \%$

AR: AUTOSAR Adaptive Framework SWC Interface

\begin{tabular}{|c|}
\hline $\begin{array}{l}\text { Total number of Equivalence Classes }\left(E_{Q C}\right)=6 \\
\text { Total number of Classes in Source } 2\left(T_{R}\right)=14 \\
\text { Semantic Similarity Metric, SSS }=\mathbf{6 / 1 4} \text { or } \mathbf{4 3} \%\end{array}$ \\
\hline F: Franca Framework SWC Interface \\
\hline
\end{tabular}

Fig.2. Evaluation of Semantic Similarity Schema (SSS) metrics for interface ontology Source 1 and Source 2. 


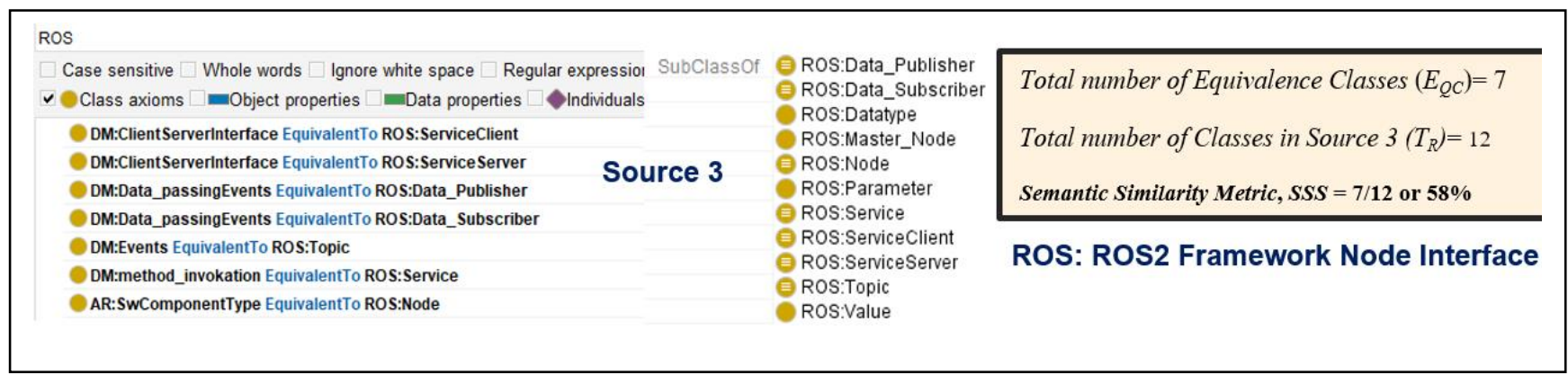

Fig.3. Evaluation of Semantic Similarity (SSS) metrics for interface ontology Source 3.

\section{Evaluation of IRR Metric for Interface Ontology Sources}

To evaluate the ABox (data) axioms (asserted or inferred by the reasoner) like sameAs and differentFrom, between the instances of the classes and subclasses of an ontology schema, we further evaluate the IRR metric for each of the given interface ontology sources (in Fig.1). As observed from Fig.4., Source 3 explicitly expresses the maximum percentage of sameAs relationships with instances of classes from other ontology sources at an instance level.

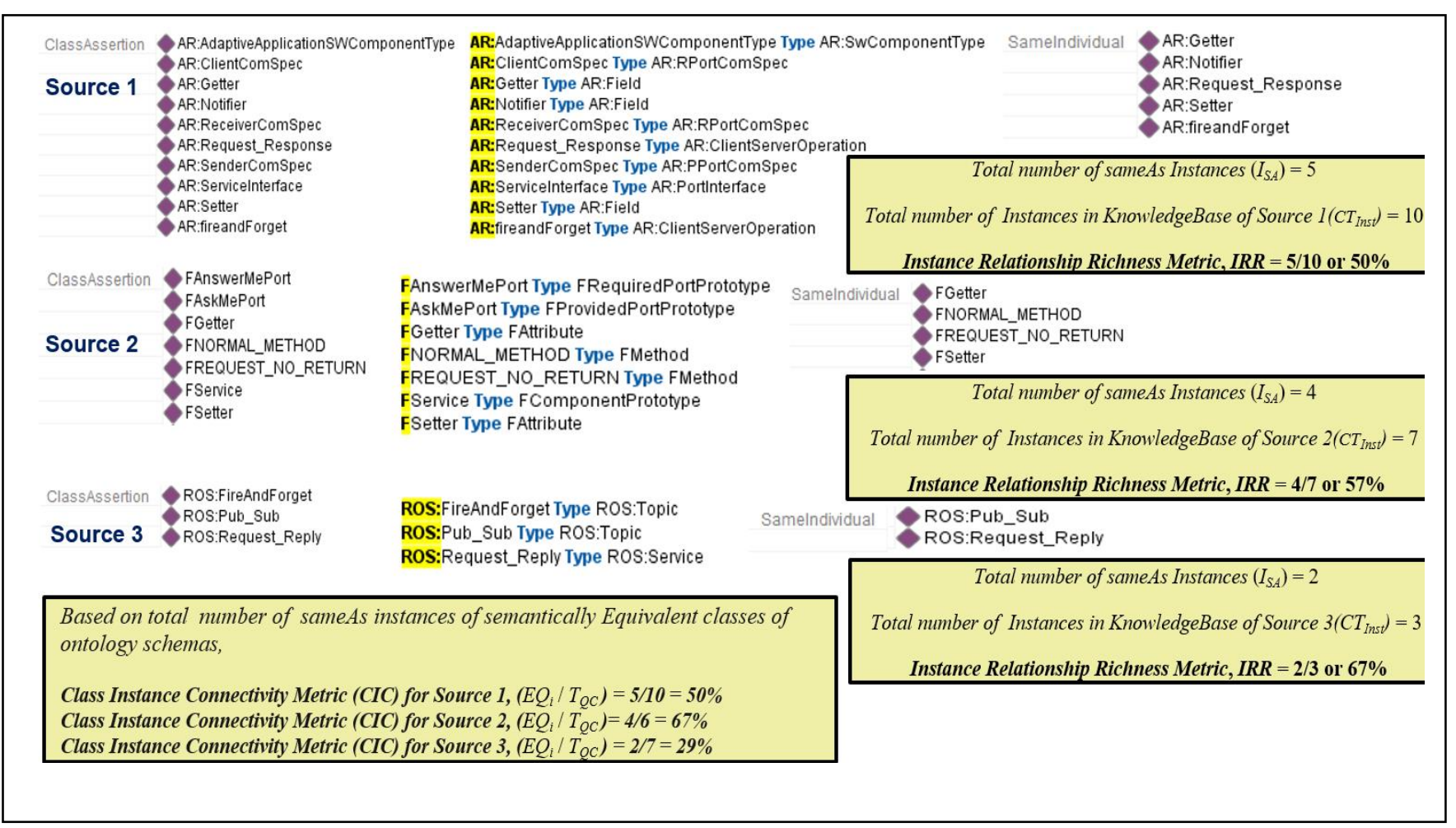

Fig.4. Evaluation of IRR and CIC Metrics for the interface ontology sources Source 1, 2 and 3.

\section{Evaluation of CIC Metric for Interface Ontology Sources}

To understand better the semantic richness of class to instance connectivity for the given interface ontological sources (in Fig.1), it is required to evaluate the $C I C$ semantic metric to understand as to how much of the semantic relationships defined between the classes in an ontology schema are actually being utilized at the instances level.
That is, measurement of percentage of sameAs instances of semantically equivalent classes for each of the given interface ontology sources (in Fig.1). As observed from Fig. 4, Source 2 expresses the maximum percentage of class to instance connectivity in the context of semantically aligned relationships. The value of CIC metric infers utilization of the knowledge modeled in the given interface ontology schemas at instance level (Tartir, Arpinar and Sheth 2010). 


\section{Validation of Interface Semantic Alignments using SPARQL}

Reasoning using SPARQL query language can be used for the validation of the semantic alignment between the concepts of given interface ontological models or sources based on inference rules. Examples of various relevant
SPARQL queries that were constructed to successfully validate the asserted and inferred axioms for the semantic alignment relationships between the various data-passing and event-driven methods classes of the given interface ontology sources (in Fig.1), at the schema level (TBox) and instance level $(A B O x)$ are illustrated in Fig. 5.

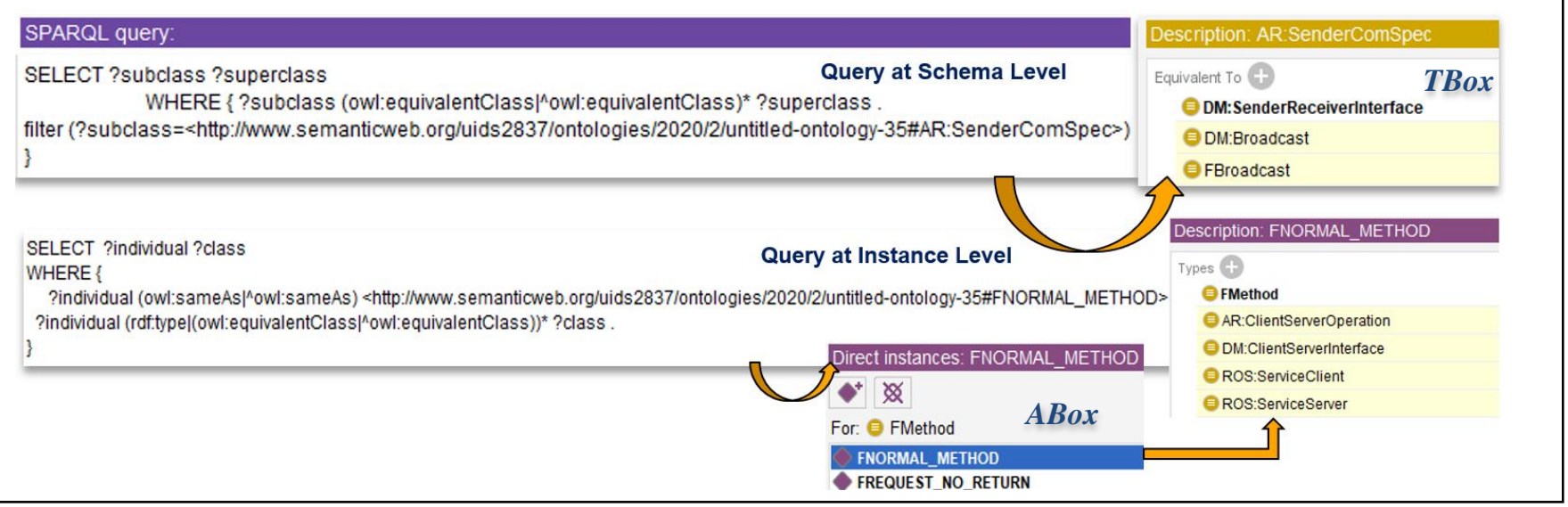

Fig.5. Overview of validation of semantic similarity relationships for interface ontology sources using SPARQL.

\section{Conclusion}

Due to the presence of a wide spectrum of distributed, heterogeneous platforms' application frameworks in vehicle domain, emerges the necessity, to evaluate and validate the quality of semantic alignments between these heterogeneous applications SWC frameworks' communication interface ontologies. This is to ensure not only semantic interoperability, correlation between these SWC frameworks but also for reusability of these SWC frameworks' interface ontologies independent of platforms. With the proposed manual evaluation approach, the semantic alignment quality metrics are evaluated based on the depth of semantic alignment relationships between the concepts of the heterogeneous SWC frameworks' interface ontologies. A vehicle domain case study has been considered for the demonstration of the evaluation and validation approach. The proposed approach reveals values of semantic alignment quality metrics, therefore must be considered in future for semantic integration of the given interface ontologies. Nevertheless, still more work is required in directions to automate the proposed evaluation approach in the future.

\section{Acknowledgement}

This work was partially supported by Ministry of Education, Youth and Sports of the Czech Republic, university specific research, project SGS-2019-018, Processing of heterogeneous data and its specialized applications.

\section{References}

Arnold, P. and Rahm, E. 2014. Enriching Ontology Mappings with Semantic Relations. In Proceedings of Data \& Knowledge Engineering. DOI: 10.1016/j.datak.2014.07.001.

Breitman, K., Felicíssimo, C. and Cysneiros, L. 2003. Semantic Interoperability by Aligning Ontologies. In Proceedings of WER03-Workshop em Engenharia de Requisitos, 213-222, Brasil.

De, S., Niklas, M., Rooney, B., Mottok, J. and Brada, P. 2019. Towards Semantic model-to-model Mapping of Cross-Domain Component Interfaces for Interoperability of Vehicle Applications: An Approach towards Synergy Exploration. In CEUR Workshop proceedings, ModComp, Vol. 2442, Germany.

Feld, M. and Müller, C. 2011. The Automotive Ontology: Managing Knowledge Inside the Vehicle and Sharing it Between Cars. In Proceedings of 3rd International Conference on Automotive User Interfaces and Interactive Vehicular.79-86.

Guarino N. and Welty, C. 2002. Evaluating ontological decisions with OntoClean. Communications of the ACM, 45(2):61-65.

Silva, J., Revoredo, K., Baião, F. and Euzenat, J. (2020). A lin : improving interactive ontology matching by interactively revising mapping suggestions. The Knowledge Engineering Review 35.

Staab, S., Walter, T., Parreiras, F.S. 2014. An ontology-based framework for domain-specific modeling. Software \& Systems Modeling 13: 83-108.

Tartir, S., Arpinar, I. B. and Sheth, A. 2010. Ontological Evaluation and Validation. In book: Theory and Applications of Ontology: Computer Applications: 115-130.

Vrandecic, D. and Sure, Y. 2007. How to design better ontology metrics. In Proceedings of the 4th European Semantic Web Conference (ESWC'07) 4519: 311-325, Springer. 